\title{
Aufsatz
}

Lukas Bittner*

\section{„Wenn Soldaten marschieren, ist es zu spät“ - Abschreckung 2.0 im Zeitalter hybrider Konflikte}

https://doi.org/10.1515/sirius-2018-2002

Kurzfassung: Die Formen der politischen Auseinandersetzungen sind laufend Veränderungen unterworfen. Carl von Clausewitz hat dies in seinem berühmten Bild des Chamäleons für den Wandel des Krieges charakterisiert. Konflikte zwischen Staaten werden heute hybrid und unterhalb der völkerrechtlich relevanten Schwelle für internationale bewaffnete Auseinandersetzungen ausgetragen. In der Folge muss sich auch die Sicherheitspolitik darauf ausrichten und das Verteidigungsdispositiv neu definieren. Abschreckung in Zeiten hybrider Konflikte heißt auch, über die militärische Komponente hinaus Maßnahmen zu entwickeln. Wenn Soldaten in Marsch gesetzt werden, um Territorien zu besetzen, ist der Konflikt in der letzten Phase. Dann ist es bereits zu spät für eine Reaktion, da zuvor die notwendigen politischen Fakten geschaffen wurden. Sicherheitspolitik muss daher hybriden Konflikten bereits im nicht-militärischen Raum wirksam begegnen.

Schlüsselwörter: Abschreckung, hybride Konflikte, hybrider Krieg, Sicherheitspolitik

Abstract: The forms of political conflict are subject to constant change. Carl von Clausewitz has characterized this in his famous image of the Chameleon for the change of war. Today, conflicts between states are hybrid and below the internationally relevant threshold for international armed conflicts. As a result, security policy must also focus on these forms of conflict and redefine the defence posture. Deterrence in times of hybrid conflicts also means developing measures beyond the military component. When soldiers are deployed to occupy territories, the conflict is in the final stage. In this phase, it is already too late for a reaction because previously the necessary political facts were created. Security policy must therefore address hybrid conflicts in a non-military area effectively.

\footnotetext{
*Kontakt: Lukas Bittner, MA, wissenschaftlicher Mitarbeiter in der Abteilung Verteidigungspolitik des Bundesministeriums für Landesverteidigung in Wien. Der Inhalt dieses Beitrages gibt die persönliche Einschätzung des Autors wieder und entspricht nicht notwendigerweise den Positionen des Bundesministeriums für Landesverteidigung. Email: lukas.bittner@bmlv.gv.at
}

Keywords: deterrence, hybrid conflicts, hybrid war, defence policy

\section{Einleitung}

Seit der Annexion der Halbinsel Krim durch die Russische Föderation im Jahr 2014 sind hybride Bedrohungen in der sicherheitspolitischen Diskussion allgegenwärtig. Vor allem die während der Krim-Besetzung medial präsenten „kleinen grünen Männchen“, also russische Spezialeinsatzkräfte an strategisch wichtigen Punkten, wurden zu einem Sinnbild dieser neuen Bedrohungen. Insbesondere baltische und osteuropäische Staaten befinden sich seit 2014 daher in einem permanenten Alarmzustand.

Nicht nur die Staaten selbst haben reagiert. In der Zwischenzeit wurde auf EU-Ebene eine „Hybrid Fusion Cell“1 eingerichtet und seit Mitte 2017 gibt es in Finnland ein „European Centre of Excellence for Countering Hybrid Threats“2. Auch die NATO hat auf die anhaltenden Spannungen mit Russland reagiert. Beim NATO-Gipfel 2016 in Warschau wurde der Beschluss zur „enhanced Forward

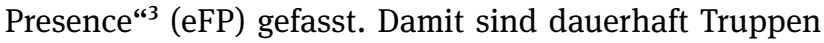
der Allianz im Baltikum beziehungsweise Osteuropa stationiert (auch wenn die Truppenteile selbst rotieren). ${ }^{4}$ Gerade russische Großübungen, wie die ZAPAD $2017^{5}$ im September, führen in einigen NATO-Staaten zu einer zunehmenden Nervosität. In vertraulichen Gesprächen mit militärischen Vertretern osteuropäischer NATO-Staaten wird immer wieder die Sorge geäußert, dass es unter Umständen zu einem Einmarsch Russlands in andere Staaten kommen könne und deswegen heute operative Pläne notwendig seien, wie man die NATO-Truppen im Notfall verstärken könne. Darüber hinaus wird eine Ver-

1 http://europa.eu/rapid/press-release_MEMO-16-1250_de.htm. 2 https://www.hybridcoe.fi/.

3 https://www.nato.int/cps/en/natohq/topics_136388.htm? selectedLocale $=$ en.

4 https://www.nato.int/nato_static_fl2014/assets/pdf/pdf_2017_ 06/20170629_170629-eFP-map-en.pdf.

5 DiePresse: Russische Groß-Kriegsspiele an der Nato-Ostflanke; 14. 09. 2017. 
stärkung der bereits vorhandenen eFP-Einheiten gefordert. ${ }^{6}$ Aber sind militärische Muskelspiele die richtige Antwort auf hybride Bedrohungen?

Die diesem Artikel zugrundeliegende Hypothese behauptet das Gegenteil. Hybriden Bedrohungen kann und muss man vor allem nicht-militärisch begegnen, denn diese agieren weit unter der Grenze einer militärischen Konfrontation und schaffen Fakten, bevor das Militär im konventionellen Sinn zum Einsatz kommt. Der russische Generalstabschef Valery Gerasimov sieht das Verhältnis von militärischen und nicht-militärischen Mitteln in der Konfliktaustragung bei 1:4. ${ }^{7}$ Achtzig Prozent der Aktivitäten finden also im nicht-militärischen Bereich statt. Militärische Maßnahmen (Übungen etc.) stellen eher eine Bedrohungskulisse beziehungsweise ein Eskalationspotenzial dar. Die - hybride - Interessensdurchsetzung selbst erfolgt nicht-militärisch. Folglich kann man argumentieren: Wenn Soldaten marschieren, ist es zu spät, denn zuvor wurden entscheidende politische Fakten geschaffen, die eine militärische Antwort sehr unwahrscheinlich werden lassen, wie die Besetzung der Krim gezeigt hat.

\section{Hybride Bedrohungen als Ausdruck eines Internationalen Systems}

Im vergangenen Jahrzehnt haben sich die Grundlagen des internationalen Systems insofern verändert, da heute die (offene) Durchsetzung nationaler Interessen wieder ins Zentrum nationaler Außenpolitiken gerückt ist ${ }^{8}$. Offensichtlichstes Beispiel ist Russland, das seit 2014 in aller Deutlichkeit hat erkennen lassen, dass es an der europäischen Friedensordnung kein Interesse mehr hat und eine Politik der Revision von Grenzen und der Destabilisierung westlicher Gesellschaften verfolgt. Aber auch US-Präsident Donald Trumps „America-First“-Politik ist ein Indikator für die zunehmende Bedeutung einer klassischen Politik der Verfolgung nationaler Interessen. Für Trump muss sich das internationale Engagement für die Vereinigten Staaten auch finanziell rechnen. ${ }^{9}$ Die idealistischen internationalen Bemühungen der Vereinten Nationen zur Durchsetzung von Menschenrechten, die in der „Respon-

6 Vgl. Shlapak/Johnson 2017.

7 Vgl. dazu die Grafik in Gerasimov 2013, 28.

8 Vgl. BMLVS 2017a, $14 \mathrm{ff}$.

9 Vgl. Rudolf 2017. sibility to Protect"10 ihren Höhepunkt fanden, sind heute in den Hintergrund gerückt. Bei der Durchsetzung nationaler Interessen geht es letztlich um die Einflussnahme von Staaten gegenüber anderen Staaten respektive deren Gesellschaften.

Auch wenn das internationale System, insbesondere die Ordnungsmodelle der Vereinten Nationen, heute geschwächt erscheinen, gewisse fundamentale Rahmenbedingungen bleiben bestehen. ${ }^{11}$ Das ius ad bellum, also der Krieg als legitimes politisches Instrument von Staaten, ist bis heute in den internationalen Beziehungen und im Völkerrecht grundsätzlich geächtet. ${ }^{12}$ Konflikte werden unterhalb einer völkerrechtlichen Schwelle ausgetragen und die offene gewaltsame Auseinandersetzung zwischen Staaten weitestgehend vermieden, auch wenn die Drohkulisse einer militärischen Eskalation immer wieder ein entscheidendes Element darstellt. Auseinandersetzungen finden eher in einer Grauzone zwischen den beiden Zuständen Krieg und Frieden statt. ${ }^{13}$ Die Begrifflichkeit der hybriden Bedrohungen versucht diese Form der Auseinandersetzungen zu beschreiben und mögliche Antworten darauf zu entwickeln.

In einer gemeinsamen Mitteilung an das Europäische Parlament und den Rat hat die Europäische Kommission „hybride Bedrohungen“ wie folgt umschrieben: „Auch wenn ,hybride Bedrohungen' unterschiedlich definiert werden und dabei immer wieder neuen Entwicklungen Rechnung getragen werden muss, geht es grundsätzlich darum, die Mischung von Zwang und Unterwanderung und von konventionellen und unkonventionellen Methoden (diplomatischer, militärischer, wirtschaftlicher oder technologischer Natur) $z u$ erfassen, auf die von staatlichen oder nichtstaatlichen Akteuren in koordinierter Weise zur Erreichung bestimmter Ziele zurückgegriffen werden kann, ohne dass jedoch die Schwelle eines offiziell erklärten Kriegs erreicht wird. Normalerweise liegt der Schwerpunkt auf der Ausnutzung von Verwundbarkeiten der Zielgemeinschaft und auf Verschleierungsstrategien zur Behinderung von Entscheidungsprozessen. Großangelegte Desinformationskampagnen und die Nutzung der sozialen Medien zur Beherrschung des politischen Diskurses oder zur Radikalisierung, Rekrutierung und Steuerung von Stellvertreterakteuren (,proxy actors') können als Vehikel für hybride Bedrohungen dienen." ${ }^{\text {44 }}$

10 Vgl. dazu: http://www.unric.org/en/responsibility-to-protect? layout=default.

11 Vgl. Hippler 2013, $43 \mathrm{ff}$.

12 Vgl. Etzersdorfer 2007, $62 \mathrm{ff}$.

13 Vgl. Barno/Bensahel 2015.

14 Gemeinsame Mitteilung an das Europäische Parlament und den Rat: Gemeinsamer Rahmen für die Abwehr hybrider Bedrohungen eine Antwort der Europäischen Union; S. 1. 
Die Definition dessen, was man sich unter hybriden Bedrohungen vorstellen soll, ist alleine schon aus dem Grunde schwierig, als der Begriff eher einen konfliktiven Umgang zweier Staaten beschreibt als eine spezifische Form einer staatlichen Interessendurchsetzung beziehungsweise eines aggressiven Verhaltens..$^{15}$ Es gibt also nicht „DIE“ hybride Bedrohung, sondern hybride Strategien ergeben sich immer aus spezifischen Situationen heraus. Einen nützlichen Ansatz zur Analyse von Hybridität bietet die Beschreibung, die im Rahmen des Projekts „Countering Hybrid Warfare: Understanding Hybrid Warfare" der NATO Multinational Capability Development Campaign (MCDC) entwickelt wurde. Dort versteht man unter „Hybrid Warfare”: „the synchronized use of multiple instruments of power tailored to specific vulnerabilities across the full spectrum of societal functions to achieve synergistic effects." ${ }^{16}$

Zum besseren Verständnis wurde von der NATO ein generisches Modell entwickelt, das die unterschiedlichen Felder darstellt, auf denen hybride Bedrohungen beziehungsweise Angriffe und Effekte erfolgen können. Diese umfassen Militär, Politik, Wirtschaft, Zivilgesellschaft, Infrastruktur und Information (auf Englisch MPECII, abgekürzt für „military, political, economic, civil, infrastructure, information") $)^{17}$. Angriffe können in jedem dieser Felder stattfinden, wobei erfolgreiche (synchronisierte) Angriffe (das MCDC-Projekt spricht hier von „synchronized attack packages“ - SAP) zu Effekten führen, die einen Einfluss auf das Funktionieren von staatlichen Funktionen, gesellschaftlicher Kohäsion, Wirtschaftstreiben, Handel etc. haben. Hierbei wird zwischen Effekten erster bis dritter Ordnung unterschieden, wobei diese Abstufung keine Gewichtung hinsichtlich ihrer Schwere darstellt. Vielmehr wird nach dem auslösenden Grund unterschieden. Ein Effekt erster Ordnung kann zu einem weiteren Effekt führen, ohne dass es einen direkten kausalen Zusammenhang zwischen dem Effekt zweiter Ordnung und der eigentlichen Attacke gibt.

\section{Angriffe finden jeden Tag statt}

Einflussnahmen auf Gesellschaften und Wirtschaftsbetriebe finden jeden Tag statt. Diese sind Teil eines faktischen Konkurrenzverhaltens von Staaten im Rahmen

15 Vgl. Bittner 2018, 178 ff.

16 MCDC Countering Hybrid Warfare Project (2017): Understanding Hybrid Warfare; S. 8.

17 Ebd. S. 9. globaler Gesellschafts- und Wirtschaftssysteme. Die besondere Herausforderung für Staaten bei der Bewältigung von hybriden Formen der Interessendurchsetzung besteht daher in dreifacher Hinsicht. Zum Ersten sind viele Angriffe grundsätzlich nicht (gesetzlich) verboten. So können zum Beispiel Desinformationskampagnen laufen, die „Fake News“ oder Verschwörungstheorien verbreiten. ${ }^{18}$ Aber auch Investitionen in strategische Infrastrukturen, wie zum Beispiel von Stromnetzen oder von Erdöl- und Erdgaslagern oder der Aufkauf von strategischen Rohstoffen, ist Teil einer globalisierten Wirtschaft. ${ }^{19}$ Zum Zweiten können Angriffe zwar illegal sein, aber die Unterscheidung zwischen kriminellen Machenschaften und strategischen Angriffen ist nicht immer möglich, wie beispielsweise bei den täglich stattfindenden Cyber-Angriffen. ${ }^{20}$ Es ist in vielen Fällen schwer zu unterscheiden, ob Angriffe im Rahmen von Phishing-Kampagnen zur persönlichen Bereicherung von kriminellen Personen beziehungsweise Gruppen dienen oder ob eine versuchte Einflussnahme auf ein politisches System beziehungsweise eine Gesellschaft vorliegt. Ganz abgesehen davon ist die Attribution in vielen Fällen nicht oder nur sehr schwer möglich. Und zum Dritten finden schließlich viele Angriffe in einem (MPECII-) Feld statt, resultieren allerdings mit einem Effekt in einem anderen (MPECII-)Feld und erfordern daher einen gesamtstaatlichen Ansatz in der Bewältigung. ${ }^{21}$

Derartige Einflussnahmen und Angriffe zu verhindern ist unmöglich. Ziel staatlicher Sicherheitspolitik muss es daher sein, sich bestmöglich auf diese Form der Auseinandersetzung vorzubereiten. Der große Unterschied zwischen den täglich stattfindenden Angriffen und Einflussnahmen auf Staaten, Wirtschaft und Gesellschaften und hybriden Strategien besteht vor allem in dem dahinterliegenden politischen Ziel. Die meisten Einflussnahmen und Angriffe erfolgen zur Erzielung eines persönlichen Machtgewinns beziehungsweise zur finanziellen Bereicherung. Hybride Angriffe haben jedoch eine Änderung der politischen Position eines Staates zum Ziel. Sie sind ein Instrument eines Staates, um einen anderen Staat zu einer bestimmten Handlung beziehungsweise deren Unterlassung zu zwingen, kurz gesagt: um Macht auszuüben. Staaten müssen ihre Sicherheitspolitik daher in mehreren Ebenen staffeln, um eine Balance zu finden. Auf der einen Seite ist ein Austausch zwischen Staaten, Wirtschaft und Gesellschaften in einer globalisierten Welt

18 Vgl. Thomas 2017.

19 Vgl. Mildner 2011.

20 http://map.norsecorp.com/\#/

21 MCDC Countering Hybrid Warfare Project (2017): Understanding Hybrid Warfare; S. 14. 
unabdingbar und muss auch weiterhin gewährleistet werden, eine Abschottungspolitik wäre nicht zielführend. Auf der anderen Seite müssen aber Einflussnahmen, die eine massive (souveränitätsgefährdende) nachteilige Auswirkung auf das Funktionieren von Staat, Wirtschaft und Gesellschaft haben können, verhindert und bekämpft werden. Die Herausforderung besteht nun darin, diese gezielten Einflussnahmen aus dem „Rauschen“ des täglichen Datenverkehrs herauszulesen.

Wie in der MCDC-Beschreibung von Hybridität festgestellt, identifizieren hybrid handelnde Akteure Vulnerabilitäten von staatlicher Verwaltung, politischen und wirtschaftlichen Systemen, von kritischer Infrastruktur und Informationsraum. Durch synchronisierte Angriffe wird versucht, Effekte durch die Ausnutzung dieser Schwachstellen zu erzielen. Diese möglichen Effekte sind daher das Herzstück und der Ausgangspunkt aller Strategien gegen hybride Angriffe.

\section{Hybriden Bedrohungen gesamt- staatlich begegnen}

Grundlage staatlicher Sicherheitspolitik zur Bewältigung von hybriden Bedrohungen kann nur ein gesamtstaatlicher Ansatz sein, der vor allem über die klassischen Sicherheitsministerien beziehungsweise -agenturen hinausgeht. Konzeptionell bedeutet das auch eine Sicherheitssektorreform in vielen europäischen Staaten. Außen-, Innen- und Verteidigungsministerien sowie Organe der Strafverfolgung alleine genügen nicht, um hybriden Bedrohungen zu begegnen; ein umfassender gesamtstaatlicher Ansatz ist notwendig. Auch andere gesellschaftliche Bereiche, wie Arbeit, Medien und Wirtschaft, müssen sich bewusst werden, dass sie Teil der staatlichen Sicherheitspolitik sind und hier Verantwortung übernehmen müssen.

Ausgangspunkt bei der Auseinandersetzung mit hybriden Bedrohungen muss eine gesamtstaatliche Risikoanalyse möglicher Verwundbarkeiten sein. Diese Analyse muss alle oben angesprochenen Bereiche umfassen und in einem gesamtstaatlichen Lagebild zusammengefasst werden. Aufgrund der spezifischen Vulnerabilitäten in den einzelnen Sektoren ist es notwendig, alle staatlichen Akteure in diese Risikoanalyse einzubinden. Gerade die klassischen Sicherheitsministerien stoßen bei der Bewertung und Analyse schnell an ihre fachlichen Grenzen. So ist es effektiver, die Vulnerabilitäten im Bereich der Volkswirtschaft, zum Beispiel der Rohstoffversorgung, durch Fachdienststellen des Wirtschaftsministeriums vornehmen zu lassen.
Auf der Basis dieser gesamtstaatlichen Risikoanalyse können dann mögliche Antworten auf hybride Bedrohungen entwickelt werden. Diese müssen aufgrund der zahlreichen möglichen Angriffsvektoren tief gestaffelt angelegt sein und können sich grundsätzlich an das Konzept der Abschreckung anlehnen.

\section{Abschreckung 2.0 - Ein Konzept des Kalten Krieges anpassen}

In der Sicherheitspolitik versteht man unter Abschreckung „das Einwirken auf den Willen eines potenziellen Aggressors, um ihn zu der politischen Entscheidung zu bewegen, auf politische Pression oder militärische Aggression zu verzichten, beziehungsweise im Fall einer bereits begonnenen Aggression die Kampfhandlungen einzustellen. Der Aggressor soll davon überzeugt werden, dass er bei seinem Angriff oder dessen Fortführung mehr verlieren als gewinnen, zumindest aber nicht die von ihm erwarteten Vorteile erlangen würde“. ${ }^{22}$ Die Theorie der Abschreckung ist eng mit der Systemkonfrontation im Kalten Krieg verbunden. Daher wird auch zwischen einer konventionellen und einer nuklearen Abschreckung unterschieden. Konzeptionell kann man Abschreckung in einen proaktiven und einen reaktiven Aspekt differenzieren. Während der proaktive Aspekt in der Stärkung der Außenwirkung eines Verteidigungsdispositivs liegt, sodass es erst gar nicht zur Aggression kommt, konzentriert sich der reaktive Aspekt vor allem auf den Aufbau einer Zweit- beziehungsweise Rückschlagsfähigkeit, sodass der Schaden für den Aggressor auf jeden Fall zu groß wird, um den Angriff fortzusetzen. In der Zeit des Kalten Krieges bedeutete dies, dass durch eine sichtbare konventionelle Abschreckung (Abschreckung durch die Vereitelung der Erfolgsaussichten - „deterrence by denial“) ein glaubhaftes Verteidigungsdispositiv dargestellt wurde. Darüber hinaus wurden durch die nukleare Abschreckung, insbesondere durch die Zweitschlagskapazität (Abschreckung durch Bestrafung - „deterrence by punishment“), die Kosten einer möglichen Aggression (beiderseitige nukleare Vernichtung) exorbitant in die Höhe geschraubt.

Wie zuvor dargestellt, ist die Mehrheit der aktuellen Bedrohungen im Rahmen der Hybridität nicht-militärischer Natur. Das Konzept der Abschreckung muss daher an diese geänderten Rahmenbedingungen angepasst werden, es muss gesamtstaatlich gedacht werden und alle zuvor angesprochenen Aspekte umfassen. Wobei die

22 Mayer/Hannemann/Meyer zum Felde 2012, 12 ff. 
Prinzipien der Theorie grundsätzlich weiter funktionieren. Gesamtstaatliche Sicherheitspolitik gegen hybride Bedrohungen muss grundsätzlich entlang dreier strategischer Linien erfolgen. Zum Ersten müssen Systeme und Gesellschaften resilient beziehungsweise robust gegen unterschiedliche Formen von Angriffen sein. Zum Zweiten braucht es aber auch Instrumente der Abschreckung. Diese müssen sich auf bestimmte souveränitätsgefährdende Effekte ausrichten und zu einer „Vereitelung der Erfolgsaussichten“ führen. Und schließlich braucht eine glaubhafte Sicherheitspolitik von Staaten oder Allianzen auch eine Abschreckung mit entsprechenden Rückschlagkapazitäten, um auf diese souveränitätsgefährdenden Effekte antworten zu können.

\subsection{Erste strategische Linie: Resiliente Gesellschaften und robuste Systeme}

Technische Entwicklungen in den vergangenen Jahrzehnten haben zu einer stör- und verletzungsanfälligen hochkomplexen Gesellschaft geführt, insbesondere im Bereich der „kritischen Infrastrukturen.“23 Gerade Angriffe in diesen Bereichen können heute ähnliche - souveränitätsgefährdende - Auswirkungen auf das Funktionieren von Staat und Gesellschaft haben, wie es klassische militärische Operationen im Rahmen eines konventionellen Kriegsszenarios gehabt hätten. Klassische Konzepte der militärischen Raumverteidigung greifen daher auch zu kurz, gerade weil, wie oben angesprochen, in den meisten Fällen zunächst unklar bleibt, wer der Angreifer ist und welche Ziele er verfolgt. ${ }^{24}$

In der Folge müssen daher Strukturen mit verminderten Verwundbarkeiten geschaffen und die Widerstandsfähigkeiten gestärkt werden. Dazu muss der Bevölkerung bewusst sein, dass Angriffe nicht ausschließlich im klassischen Sinne durch „hard power“-Instrumente erfolgen, sondern meistens durch „,soft power“ ${ }^{25}$ Wenn sich Gesellschaften bewusst sind, dass ihre Kohäsion das Ziel von Angriffen darstellt, können sie diese auch effektiv zurückweisen. Die Resilienz von Gesellschaften ergibt sich also aus einem präventiven Ansatz, der vor allem im Bildungssystem (in Österreich betrifft dies vor allem die „Geistige

23 Vgl. dazu das österreichische Programm zum Schutz kritischer Infrastrukturen (APCIP). Abzurufen unter: https://www.bundeskanzleramt.gv.at/schutz-kritischer-infrastrukturen.

24 Vgl. Münkler/Wassermann 2012, 80.

25 „hard power“ beziehungsweise „soft power“ werden in diesem Zusammenhang im Sinne von Joseph Nye verstanden. Kurz zusammengefasst in: Nye, Joseph: „Propaganda Isn't the Way: Soft Power.“ International Herald Tribune, 10. Januar 2003.
Landesverteidigung“26) und in der Zivilgesellschaft verankert werden muss. Eine Gesellschaft, die sich ihrer Vulnerabilitäten und des Faktums der täglich stattfindenden Angriffe bewusst ist, reagiert gelassener und effektiver auf Angriffe, wie zum Beispiel „Fake News“ und andere Desinformationskampagnen. ${ }^{27}$ Gleichzeitig müssen zentrale kritische Infrastrukturen, die für das Funktionieren von Staat und Gesellschaften fundamental sind, robust ausgestaltet werden. Dies betrifft unter anderem einen verbesserten Schutz gegen Angriffe aus dem Cyber-Raum oder Sicherheits- und Überwachungseinrichtungen an den Objekten selbst sowie diversifizierte Lager- und Transportsysteme. Wie bereits oben beschrieben, finden Angriffe auf diese Systeme täglich statt. Sie müssen daher in der Lage sein, diese Angriffe abzuwehren, sodass diese keine durch den Angreifer gewünschten Effekte erzielen.

\subsection{Zweite strategische Linie: Abschreckung durch die Vereitelung von Erfolgsaussichten}

Die klassische Form der konventionellen Abschreckung fordert ein militärisches Verteidigungsdispositiv, das dazu geeignet ist, einen möglichen Angreifer davon abzubringen, überhaupt einen Waffengang zu wagen, da der militärische Erfolg unwahrscheinlich erscheint (,deterrence by denial“). Damit ist diese Form der konventionellen Abschreckung durch eine Vereitelung der (militärischen) Erfolgsaussichten geprägt. Da in hybriden Bedrohungsszenarien militärische Waffengänge jedoch eher eine Drohkulisse darstellen, wird die Einflussnahme in anderen Politikfeldern vorgenommen. Ein militärisches Verteidigungsdispositiv alleine als konventionelle Abschreckung ist daher nicht ausreichend, gerade weil Aggressoren in hybriden Konflikten versuchen werden, unterhalb der völkerrechtlichen Schwelle einer militärischen Auseinandersetzung zu bleiben.

Die Erweiterung der Abschreckung durch die Vereitelung von Erfolgsaussichten auf andere - nicht-militärische - Bereiche ist daher geboten. Aufbauend auf einer Vulnerabilitätsanalyse kann dann ein hybrides Verteidigungsdispositiv erstellt werden. Im wirtschaftlichen Bereich könnte dieses Verteidigungsdispositiv zum Beispiel legislative Maßnahmen umfassen, wonach ausländische Beteiligungen an Unternehmen kritischer Infrastrukturen zentral erfasst werden müssen. In dieser

26 Vgl. dazu: https://www.bmb.gv.at/schulen/unterricht/ba/glv. html.

27 Vgl. Thomas 2017. 
Hinsicht könnten auch Regelungen erlassen werden, die ausländische Beteiligungen an Firmen von strategischer Relevanz über einen bestimmten Anteil hinaus verbieten beziehungsweise die eine Not-Verstaatlichung ermöglichen. Hinsichtlich der Versorgung mit strategischen Rohstoffen wären Diversifizierung und Rohstoffpartnerschaften eine mögliche Form der Abschreckung. ${ }^{28}$ Welche Nachteile eine einseitige Versorgung mit Rohstoffen mit sich bringen kann, zeigt sich immer wieder in der Frage der russischen Gaslieferungen nach Europa beziehungsweise deren Unterbrechungen. ${ }^{29}$ Eine Abschreckung durch die Verweigerung der Erfolgsaussichten würde durch eine Diversifizierung der Versorgungslinien und nachhaltige strategische Reserven, einschließlich der Möglichkeit einer gegenseitigen Belieferung, erreicht werden.

Gerade der Informationsraum spielt eine zentrale Rolle in hybriden Konflikten. Hierbei geht es in den meisten Fällen um die Entwicklung eines Narrativs, das den eigenen Standpunkt unterstützt. Legislative Maßnahmen sind in diesem Bereich jedoch sensibel. Die Grenze zwischen einer freien Berichterstattung - als Grundprinzip liberaler Gesellschaften - und bewussten Desinformationskampagnen ist schmal. Über rechtliche Bestimmungen hinausgehend ist daher eine Stärkung des zivilgesellschaftlichen Diskurses wichtig. Die Diversität des modernen Medienangebots hat für die freie Meinungsbildung große Vorteile, gleichzeitig ist eine mögliche Beeinflussung und Kontrolle der multimedialen „Konfiguration“ einer Gesellschaft auch heute ein zentraler Machtfaktor. ${ }^{30}$ Geboten wären daher Maßnahmen nach dem Prinzip des „naming, blaming \& shaming“"31 - also Instrumente der strategischen Kommunikation, ${ }^{32}$ die diese Kampagnen identifizieren und ihnen aktiv gegensteuern, sodass der durch den aggressiven Akteur - gewünschte Effekt nicht eintritt.

\subsection{Dritte strategische Linie: Abschreckung durch Bestrafung}

Auch die Abschreckung durch Bestrafung (,deterrence by punishment“) muss erweitert gedacht werden. In der klassischen Theorie dachte man in dieser Hinsicht insbeson-

$28 \mathrm{Vgl}$. Steinbach/Buchholz 2012, $26 \mathrm{ff}$.

29 Vgl. Westphal 2009, $18 \mathrm{ff}$.

30 Vgl. Dengg/Schurian 2016, 65.

31 Libby Purves: Let's name and shame the fake news bandits; The Times, 13. 02. 2017.

32 Vgl.: Bericht über das Thema „Strategische Kommunikation der EU, um gegen sie gerichtete Propaganda von Dritten entgegenzuwirken“ (2016/2030[INI]). dere an die Nuklearfähigkeiten von Verteidigungsbündnissen. „Kernwaffen sind neben konventionellen Kräften und Raketenabwehrfähigkeiten ein zentraler Bestandteil der Gesamtfähigkeiten der NATO zur Abschreckung und Verteidigung. Die Überprüfung hat gezeigt, dass das Dispositiv der nuklearen Kräfte des Bündnisses gegenwärtig die Kriterien eines wirksamen Abschreckungs- und Verteidigungsdispositivs erfüllt. “33 So steht es im NATO Deterrence and Defence Posture Review (DDPR). Und dieses führt weiter aus, dass die NATO ein nukleares Bündnis bleibt, solange es Kernwaffen gibt. ${ }^{34}$

Im Rahmen hybrider Konflikte ist allerdings nukleare Abschreckung durch Bestrafung nicht zielführend. Die Grundidee der nuklearen Abschreckung bleibt ein mögliches nukleares Armageddon, welches allerdings nur durch die existenzielle Schwere des Angriffs seine Rechtfertigung finden kann. Hybride Konflikte sind hingegen Auseinandersetzungen, die grundsätzlich nicht mit offenen militärischen Mitteln unterhalb der Schwelle eines internationalen bewaffneten Konfliktes ausgetragen werden. Die Eskalation der nuklearen Annullierung geht in der Regel weit über jedes - in diesem Zusammenhang denkbare Szenario hinaus.

Gefragt sind vielmehr nicht-militärische Formen der Abschreckung durch Bestrafung. Es gibt bereits Formen, die angepasst angewendet werden könnten, wie die der Finanz-, Wirtschafts- und politischen Sanktionen, wie sie durch die USA ${ }^{35}$, die $\mathrm{EU}^{36}$ und Russland ${ }^{37}$ gegeneinander im Zuge der Krim-Krise erlassen wurden. Die Effektivität dieser Sanktionen ist umstritten und gerade in der Europäischen Union gibt es immer wieder Stimmen, die eine Aufhebung beziehungsweise Flexibilisierung dieses Sanktionsregimes gegen Russland fordern..$^{38}$ Ein anderer Ansatz der wirtschaftspolitischen Abschreckung durch

33 Kapitel II, Pkt. 8 der deutschen Übersetzung des Deterrence and Defence Posture Review (DDPR) auf der Internetseite der deutschen NATO-Vertretung in Brüssel: http://www.nato.diplo.de/contentblob/3528424/Daten/2431953/ChicagoPostureReviewdtDLD.pdf.

34 Zur Nuklearstrategie der NATO vgl. Kamp 2017, 359 ff.

35 Vgl. dazu die Seite des US-Außenministeriums: https://www. state.gov/e/eb/tfs/spi/ukrainerussia/.

36 Vgl. dazu die Verordnungen der Europäischen Union; http://eurlex.europa.eu/legal-content/DE/TXT/PDF/?uri=CELEX:32014R0208 \&rid=7; beziehungsweise http://eur-lex.europa.eu/legal-content/DE/ TXT/PDF/?uri=CELEX:32014R0269.

37 Vgl. dazu die Listen bezüglich des russischen Importverbots von Lebensmitteln: http://www.gtai.de/GTAI/Navigation/DE/Trade/Maerkte/Special/russland-sanktionen, $\mathrm{t}=21$-einfuhrverbot-fuer-lebensmittel-aus-der-eu,did=1260588.html und von Personen: http://www.gtai. de/GTAI/Content/DE/Trade/_SharedDocs/Pdf/Maerkte/RusslandSanktionen/visasperrliste-einreiseverbote-russland.pdf?v=2 38 Vgl. Fischer 2017. 
Bestrafung wäre die Abkoppelung einzelner Staaten von Finanztransaktionssystemen (zum Beispiel dem SWIFT). Auch diese Maßnahmen waren gegenüber Russland angedacht. ${ }^{39}$ Diese Transaktionssysteme stellen zentrale Nervenbahnen der globalisierten Weltwirtschaft dar und eine Trennung würde verheerende Auswirkungen haben. Es stellt sich heute jedoch die Frage, wie lange diese Androhung noch wirksam sein könne, da es in diesem Bereich durch den Aufbau alternativer Systeme zu einer Diversifizierung kommt. ${ }^{40}$

Im Bereich der Sanktionsregime werden immer wieder die wirtschaftlichen Kosten als Gegenargumente ins Treffen geführt. Hier muss jedoch festgestellt werden, dass staatliche Zwangsmaßnahmen immer mit Kosten verbunden sind. Eine Eskalation zu einer konventionellen militärischen Konfrontation oder gar zu einem Nuklearkrieg würde einen exorbitanten Tribut hinsichtlich wirtschaftlicher Ressourcen, aber vor allem hinsichtlich der betroffenen Menschen fordern, der in keinem Verhältnis zu den Kosten von Sanktionen in den Bereichen Politik, Finanzen und Wirtschaft steht.

Ein weiteres Potenzial zur Entwicklung von Maßnahmen zur Abschreckung durch Bestrafung findet sich derzeit im Bereich des Informationsumfelds. Gerade der Cyber-Raum hat in den vergangenen Jahren massiv an Bedeutung gewonnen. Wie bereits festgestellt wurde, finden Angriffe auf staatliche und private IT-Systeme täglich statt. In der Diskussion stellt sich nun die Frage, wie man diesen Angriffen am besten begegnet. Während wie oben beschrieben - die Robustheit von Systemen gestärkt werden muss, ist auch der Aufbau eines Abschreckungspotenzials notwendig. Gerade strategisch und groß angelegte Cyber-Angriffe auf kritische Infrastrukturen im Rahmen von hybriden Konflikten müssen daher zum Teil mit offensiven Maßnahmen beantwortet werden, um einen Angriff beenden zu können. Gerade diese offensiven Fähigkeiten sind ein wesentlicher Bestandteil einer Abschreckung durch Bestrafung.

\section{Zusammenfassung}

Die Formen der aggressiven politischen Auseinandersetzungen sind einem laufenden Wandel unterworfen. Clausewitz hat dies in seinem berühmten Bild des Chamäleons für den Wandel der Konfliktformen charakterisiert. Auch

39 Vgl. Vanessa Steinmetz: Europas Geheimwaffe gegen den Kreml, SPIEGEL-Online, 1. September 2014.

40 Vgl. Lohmann 2014. wenn sich die heutige internationale Ordnung eher in einer (Welt-)Unordnung darstellt ${ }^{41}$ und das völkerrechtliche Gewaltverbot in einigen Fällen unterlaufen wird, so sind die grundsätzlichen Rahmenbedingungen weiterhin gültig. Der Krieg - im Sinne einer bewaffneten Auseinandersetzung zwischen Staaten - ist weiterhin kein legitimes Mittel staatlicher Politik. Allerdings hat sich der Umgang zwischen Staaten in vielen Fällen verschärft. Konflikte zwischen Staaten werden hybrid und unterhalb der völkerrechtlich relevanten Schwelle für konventionelle militärische Konflikte ausgetragen. In der Folge muss sich auch die Sicherheitspolitik auf diese Konfliktformen ausrichten und das Verteidigungsdispositiv neu definieren.

In Österreich wird derzeit über eine Reaktivierung der „Umfassenden Landesverteidigung“ (Artikel 9a des Bundesverfassungsgesetzes) diskutiert. ${ }^{42}$ Dieses Konzept aus dem Kalten Krieg soll auf die sicherheitspolitischen Bedrohungen und Herausforderungen der heutigen Zeit angepasst werden. Im Unterschied zu vielen anderen Verteidigungskonzepten fußt die „Umfassende Landesverteidigung “ auf vier - zumindest theoretisch - gleichwertigen Säulen. Neben der militärischen gibt es noch die zivile, die wirtschaftliche und die geistige Landesverteidigung. Grundsätzlich hat dieses Konzept das Potenzial, gegen hybride Bedrohungen wirksam zu sein - wobei Anpassungen notwendig sind, da das ursprüngliche Konzept auf einen Kriegsfall ausgerichtet war und relativ wenige präventive beziehungsweise proaktive Maßnahmen gesetzt wurden. Die Anpassungen müssten an dieser Stelle in Richtung eines unterschwelligen Konfliktzustandes vorgenommen werden.

Abschreckung in Zeiten hybrider Konflikte heißt auch, über die militärische Komponente hinaus Maßnahmen zu entwickeln. Das Grundprinzip der hybriden Konflikte ist, unterhalb der Schwelle einer militärischen Konfrontation zu bleiben, auch wenn das militärische Eskalationspotenzial ein wesentliches Druckmittel ist. Wenn Soldaten in Marsch gesetzt werden, um Territorien zu besetzen, ist der Konflikt in der letzten Phase. In dieser ist es bereits zu spät für eine Reaktion, da zuvor die notwendigen politischen Fakten geschaffen wurden. Sicherheitspolitik muss daher hybriden Konflikten bereits im nicht-militärischen Raum schlagkräftig und wirksam begegnen.

41 Masala 2016.

42 Vgl. BMLVS 2017b, 28. 


\section{Literatur}

Barno, David/Bensahel Nora (2015): Fighting and Winning in the „gray zone“, War on the Rocks, https://warontherocks. com/2015/05/fighting-and-winning-in-the-gray-zone/.

Bittner, Lukas (2018): Kein Frieden und noch kein Krieg: „Hybride Konflikte“ als Form der staatlichen Interessendurchsetzung Oder: Warum der Kampf gegen den sogenannten „Islamischen Staat“ kein hybrider Konflikt ist, Österreichische Militärische Zeitschrift, 56 (2), 178-187.

BMLVS 2017a: Direktion für Sicherheitspolitik: Sicher. Und Morgen? - Sicherheitspolitische Jahresvorschau 2018. Wien: Bundesministerium für Landesverteidigung und Sport.

BMLVS 2017b: Positionspapier des Generalstabs zur Weiterentwicklung der Landesverteidigung: „Sicherheit verbessern - Bundesheer stärken“. Wien: Bundesministerium für Landesverteidigung und Sport.

Dengg Anton/Schurian Michael, Hrsg. (2015): Vernetzte Unsicherheit - Hybride Bedrohungen im 21. Jahrhundert. Heft 15/2015 der Schriftenreihe der Landesverteidigungsakademie. http://www.bundesheer.at/pdf_pool/publikationen/lvak_ sr_15_2015_hybride_bedrohung_dengg_schurian.pdf

Etzersdorfer, Irene (2007): Krieg - Eine Einführung in die Theorie bewaffneter Konflikte. Wien: Böhlau/UTB.

Fischer, Sabine, Hrsg. (2017): Sanktionen als Dauerzustand? Vorschlag für eine Flexibilisierung der EU-Sanktionspolitik gegenüber Russland. Berlin: Stiftung Wissenschaft und Politik.

Gerasimov, Valery (2013): The Value of Science is in the Foresight, Übersetzt aus dem Russischen durch Robert Coalson; abgerufen unter: https://euagenda.eu/publications/ new-challenges-demand-rethinking-the-forms-and-methodsof-carrying-out-combat-operations-general-of-the-army-valerygerasimov-chief-of-the-general-staff-of-the-russian-federationarmed-forces.

Hippler, Jochen (2013): Schlaglicht - Der Aufstieg neuer Mächte, internationale Kooperationsformen und die Reaktion der Industrieländer. In: Debiel, Tobias / Hippler, Jochen/ Roth, Michèle/ Ulbert, Cornelia, Hrsg.: Globale Trends - Frieden Entwicklung Umwelt. Frankfurt a.M.: Fischer.
Kamp, Karl-Heinz (2017): Eine nukleare Neuausrichtung der NATO. In: SIRIUS - Zeitschrift für Strategische Analysen, 1 (4), 359-366

Lohmann, Sascha, Hrsg. (2014): Minenfelder der US-Außenwirtschaftspolitik: unilaterale Finanzsanktionen im Dienst nationaler Sicherheit. Berlin: Stiftung Wissenschaft und Politik.

Masala, Carlo (2016): Weltunordnung: Die globalen Krisen und das Versagen des Westens. München: C. H. Beck.

Meier, Ernst-Christoph/ Hannemann, Andreas/ Meyer zum Felde, Rainer, Hrsg. (2012): Wörterbuch zur SicherheitspolitikDeutschland in einem veränderten internationalen Umfeld. 8. Auflage. Hamburg: E.S. Mittler.

Mildner, Stormy-Annika, Hrsg. (2011): Konfliktrisiko Rohstoffe? Herausforderungen und Chancen im Umgang mit knappen Ressourcen. Berlin: Stiftung Wissenschaft und Politik.

Münkler Herfried/Wassermann Felix (2012): Von strategischer Vulnerabilität zu strategischer Resilienz - Die Herausforderung zukünftiger Sicherheitsforschung und Sicherheitspolitik. In: Gerhold, Lars/ Schiller, Jochen, Hrsg.: Perspektiven der Sicherheitsforschung. Beiträge aus dem Forschungsforum Öffentliche Sicherheit. Hamburg: Peter Lang, 77-95.

Rudolf, Peter (2017): US-Außenpolitik unter Präsident Trump. Berlin: Stiftung Wissenschaft und Politik.

Shlapak, David A./Johnson, Michael W. (2017): Reinforcing Deterrence on NATO's Eastern Flank. St. Monica, Cal.: RAND Corporation.

Steinbach Volker/Buchholz Peter (2012): Sicherung der Rohstoffversorgung für die Zukunft des Technologiestandortes Deutschland, ACAMONTA - Zeitschrift für Freunde und Förderer der TU Bergakademie Freiberg, 18 (1), 26-29.

Thomas, Timothy (2017): Russia's 21st century information war: working to undermine and destabilize populations, Defence Strategic Communications, 1 (2), 10-25.

Westphal, Kirsten, Hrsg. (2009): Russisches Erdgas, ukrainische Röhren, europäische Versorgungssicherheit: Lehren und Konsequenzen aus dem Gasstreit 2009. Berlin: Stiftung Wissenschaft und Politik. 